RESEARCH ARTICLE

\author{
M. Filipowicz • M. Kępczyk
}

\title{
A note on zero-divisors of commutative rings
}

Received: 13 May 2011 / Accepted: 3 November 2011 / Published online: 11 April 2012

(C) The Author(s) 2012. This article is published with open access at Springerlink.com

Abstract In this paper we show that if a ring $R$ has finite Goldie dimension, then every finitely generated ideal of $R$ consisting of zero-divisors has non-zero annihilator. We also construct an example of a ring of infinite Goldie dimension such that above condition does not hold.

Mathematics Subject Classification (2010) 16P60 - 16D25

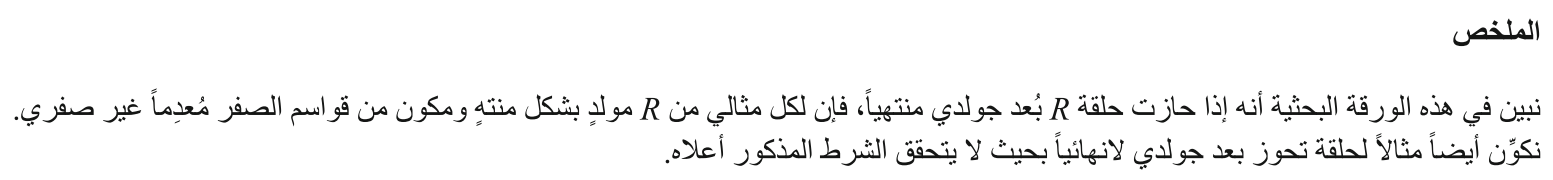

\section{Introduction}

Different algebraic systems play a substantial role in studies of some problems of analysis. One can find some examples of them in [2,3]. Such systems reflect selected properties of analytic objects. Their properties, studied with use of algebraic tools, can be applied to infer properties of the analytic objects. The motivations for the studies presented in this paper come from some problems of control theory. In [2,3] Bartosiewicz used the ring of distributions $K$ (cf. [3], p. 299) in studies of solution of some functional-differential equations. For that purpose, he studied zero-divisors of the ring $K$.

Recall that $a \in R$ is a zero-divisor of a commutative ring $R$ if and only if there is a nonzero element $b \in R$ such that $a b=0$. A zero-divisor is called proper if it is not equal to 0 .

For a subset $X$ of $R$, the annihilator of $X$ in $R$ is defined as $a n_{R}(X)=\{a \in R \mid a X=X a=0\}$. Clearly $a \in R$ is a zero-divisor if and only if $a n_{R}(a) \neq 0$.

Bartosiewicz proved that the zero-divisors of the ring $K$ form an ideal of this ring. He obtained this as a consequence of the following more general property which is satisfied by the ring $K$ :

Proposition 1.1 If a commutative ring $R$ satisfies the condition:

$(\star)$ every finite set of zero-divisors of $R$ has a nonzero annihilator then the set of zero-divisors of $R$ is an ideal of the ring $R$.

M. Filipowicz · M. Kȩpczyk ( $\varangle)$

Department of Mathematics,

Bialystok University of Technology,

Wiejska 45 A, 15-351 Białystok, Poland

E-mail: m.kepczyk@pb.edu.pl

M. Filipowicz

E-mail: mfilipowicz@kki.net.pl 
Bartosiewicz asked (private communication), whether the converse holds, i.e. $(\star)$.

(P1) If the set of zero-divisors of a commutative ring $R$ is an ideal of the ring $R$ then $R$ satisfies the condition

The answer to the above question is negative, because there are examples of rings with ideals consisting entirely of zero-divisors and such that the ideals contain finite subsets with zero annihilator. General examples of such rings are presented in $[7,16]$. In Sect. 3 we give one more, as a simpler and better illustration of the situation considered in this paper.

It is well-known (cf. [1]) that the set of non-units of a commutative ring is an ideal if and only if the ring is local (i.e. has a unique maximal ideal). It seems that obtaining of similar characterization for zero-divisors is difficult. In this paper, we reduce that problem to the following question: for which classes of rings the condition $(\star)$ is satisfied? The most natural class of rings satisfying this condition is the class of Noetherian rings. It is well-known that if $I$ is an ideal in a Noetherian ring and if $I$ consists of zero-divisors, then the annihilator of $I$ is non-zero. Therefore, zero-divisors form an ideal in the class of Noetherian rings. In Sect. 3, we generalize that fact to the class of rings with finite Goldie dimension. Precisely, we show that the condition $(\star)$ holds for each proper ring with finite Goldie dimension (Theorem 3.4). For different classes of rings, condition ( $\star$ ) was considered by other authors, e.g. $[8,11,15]$. In our paper the obtained result concerns the class of rings that was never examined before. Moreover, in many cases, it is not difficult to verify whether a ring has a finite Goldie dimension. Therefore, Theorem 3.4 is a useful tool for studying the condition ( $\star$ ). For example, this theorem can be applied to the ring of distributions $K$ mentioned above for which the Goldie dimension is finite.

The related topics to the one examined in this paper one can find in $[4,9,12-14]$.

\section{Preliminaries}

All rings in this paper are associative and commutative but we do not assume that each ring has an identity element. To denote that $I$ is an ideal of a ring $R$ we write $I \triangleleft R$. For undefined terms and used facts we refer the reader to $[1,10]$.

Note that if the set of zero-divisors of a ring $R$ forms an ideal $I$, then $I$ is a ring without identity consisting of zero-divisors. Thus it is quite natural to consider rings without identity.

It is easy to check that the (P1) is equivalent to the following question.

(P2) Is it true that the annihilator of every finite set is non zero for every commutative ring consisting of zero-divisors?

Now we recall some notions and a result, which will be used later.

An ideal $I$ of a ring $R$ is called essential if for every non-zero ideal $J$ of $R, I \cap J \neq 0$.

A non-zero ideal $I$ of a ring $R$ is called uniform if every nonzero ideal of $R$ contained in $I$ is essential in $I$.

A ring $R$ is said to have finite Goldie dimension if it does not contain infinite direct sums of non-zero ideals.

It is well-known (cf. [5,6]) that a ring $R$ has a finite Goldie dimension if and only if it contains a direct sum $I=I_{1} \oplus \cdots \oplus I_{n}$ of uniform ideals $I_{i}$ and $I$ is an essential ideal of $R$.

It is clear that Noetherian rings have a finite Goldie dimension.

\section{Results}

In this section we will construct a ring with infinite Goldie dimension, giving a negative answer to (P2). We also show that $(\mathbf{P 2})$ has a positive answer for rings with finite Goldie dimension.

Suppose that $R$ is a ring and $M$ is a left and right $R$-module. $M$ is called $R$-bimodule if for arbitrary $a, b \in R$ and $m \in M, a(m b)=(a m) b$.

If $R$ is a ring and $V$ is an $R$-bimodule, then the set

$$
\left\{\left(\begin{array}{ll}
r & v \\
0 & r
\end{array}\right) \mid r \in R, v \in V\right\}
$$

is a ring with respect to canonical matrix addition and multiplication.

Example 3.1 Let $P=F[x, y]$ be the polynomial ring in two commutative variables $x, y$ over a field $F$ and let $A=x P+y P$. Clearly, $A$ is a commutative ring (without identity) and for every $w \in A, w P \triangleleft P$ and 
$P / w P$ has a natural structure of $P$ - bimodule. Let $N$ be the $P$-bimodule $\bigoplus_{0 \neq w \in A} P / w P$. Obviously $N$ is also $A$-bimodule so we can form the ring $R=\left\{\left(\begin{array}{cc}a & n \\ 0 & a\end{array}\right) \mid a \in A, n \in N\right\}$.

Note that for arbitrary $w \in A$ and $n \in N,\left(\begin{array}{cc}w & n \\ 0 & w\end{array}\right)\left(\begin{array}{ll}0 & e_{w} \\ 0 & 0\end{array}\right)=\left(\begin{array}{ll}0 & 0 \\ 0 & 0\end{array}\right)$, where $e_{w}$ is the element of $N$, whose $w$ th component is equal to 1 and all other components are equal to 0 . Thus $R$ consists of zero-divisors.

Now we will show that $\operatorname{ann}_{R}\left(\left\{\left(\begin{array}{ll}x & 0 \\ 0 & x\end{array}\right),\left(\begin{array}{ll}y & 0 \\ 0 & y\end{array}\right)\right\}\right)=0$. Indeed, take $\left(\begin{array}{ll}a & n \\ 0 & a\end{array}\right) \in \operatorname{ann}_{R}\left(\left\{\left(\begin{array}{ll}x & 0 \\ 0 & x\end{array}\right)\right.\right.$, $\left.\left.\left(\begin{array}{ll}y & 0 \\ 0 & y\end{array}\right)\right\}\right)$. Then $a x=a y=0$, so $a=0$. Moreover $x n=y n=0$. This means that for every $0 \neq w \in A$, $x n_{w}=y n_{w}=0$, where $n_{w}$ denotes the $w$-component of $N$. However each $n_{w}$ is of the form $p_{w}+w P$ for some $p_{w} \in P$. Since $x n_{w}=y n_{w}=0$ we get that $x p_{w} \in w P$ and $y p_{w} \in w P$. Let $x p_{w}=w p_{1}$ and $y p_{w}=w p_{2}$ for some $p_{1}, p_{2} \in P$. Then $x y p_{w}=y x p_{1}=x w p_{2}$, so $y p_{1}=x p_{2}$. This implies that $p_{1}=x p_{1}^{\prime}$ and $p_{2}=y p_{2}^{\prime}$ for some $p_{1}^{\prime}, p_{1}^{\prime} \in P$. Consequently, $x p_{w}=w x p_{1}^{\prime}$, so $p_{w}=w p_{1}^{\prime}$. This however means that $p_{w}+w P=w P$, so each component of $n$ is equal to 0 . Therefore $n=0$ which shows that $\left(\begin{array}{ll}a & n \\ 0 & a\end{array}\right)=0$ and we are done.

One easily sees that the above constructed ring $R$ contains the infinite direct sum $\bigoplus_{0 \neq w \in A}\left(\begin{array}{ll}0 & P / w P \\ 0 & 0\end{array}\right)$ of ideals, i.e., the Goldie dimension of $R$ is infinite.

It is not hard to find an example of non-Notherian ring of finite Goldie dimension.

Let $F$ be a field and let $X \cup\{\theta\}$ be a set of symbols $\left\{x_{\alpha}\right\}$, where $\alpha$ is an element of the set of positive real number with zero. Multiplication in $\mathrm{X}$ is defined as

$$
\begin{gathered}
x_{\alpha} x_{\beta}=x_{\alpha+\beta} \text { if } \alpha, \beta>0 \\
x_{0} x_{\alpha}=\theta=x_{\alpha} x_{0} \text { if } \alpha \geq 0 \\
\theta x_{\alpha}=\theta=x_{\alpha} \theta .
\end{gathered}
$$

Under this operation the set $X \cup\{\theta\}$ is a commutative semigroup. Then, let $P=F_{0}[X]$ be the contracted semigroup algebra of $X$ over $F$. It is clear that $P$ has a finite Goldie dimension. In order to prove that $P$ is non-Noetherian, it is enough to take the elements $x_{\alpha}$ for $\alpha>0$ and consider the ideals $I_{\alpha}=x_{\alpha} P$.

Now we will show that the condition $(\star)$ holds for each proper ring with finite Goldie dimension. We will need the following two lemmas which are in fact known. The latter one is just a very classical result, which one can find in [1], the former is not so classical but also known. We include their simple proofs for completeness.

Recall that an ideal $I$ of a ring $R$ is called prime if for arbitrary elements $x, y \in R \backslash I, x y \notin I$.

Lemma 3.2 If $I$ is a uniform ideal of a ring $R$ and $J$ is an ideal of $R$ then $\bar{I}_{J}=\{x \in J \mid$ for some $0 \neq y \in I, x y=0\}$ is a prime ideal of $J$.

Proof For every $a \in \bar{I}_{J} \operatorname{ann}_{R}(a) \cap I \neq 0$. Since $I$ is a uniform ideal of $R$, for arbitrary $0 \neq x, y \in \bar{I}_{\underline{J}}$, $T=\operatorname{ann}_{R}(x) \cap \operatorname{ann}_{R}(y) \cap I \neq 0$. Hence $T(x+y)=0$, so $x+y \in \bar{I}_{J}$. It is clear that for arbitrary $x \in \bar{I}_{J}$ and $r \in J, x r \in \bar{I}_{J}$. Consequently, $\bar{I}_{\underline{J}}$ is an ideal of $J$.

Suppose that $x, y \in J$ and $x y \in \bar{I}_{J}$. Then there exists $0 \neq z \in I$ such that $z x y=0$. If $z x=0$ then $x \in \bar{I}_{J}$. If $z x \neq 0$, then, since $z x \in I, y \in \bar{I}_{J}$. These show that the ideal $\bar{I}$ is prime.

Lemma 3.3 If $I_{1}, \ldots, I_{n}$ are prime ideals of a ring $R$ and $R=I_{1} \cup \cdots \cup I_{n}$, then $R=I_{i}$ for some $1 \leq i \leq n$.

Proof We can assume that the union $I_{1} \cup \cdots \cup I_{n}$ is irredundant and then we have to show that $n=1$. Suppose that $n \geq 2$. Take for arbitrary $1 \leq i \leq n, x_{i} \in I_{i} \backslash\left(I_{1} \cup \cdots \cup I_{i-1} \cup I_{i+1} \cup \cdots \cup I_{n}\right)$ and set $x=x_{1}+x_{2} \cdots \cdots x_{n}$. Since $x_{2}, \cdots, x_{n} \notin I_{1}$ and $I_{1}$ is a prime ideal, $x_{2} \cdots x_{n} \notin I_{1}$, so $x \notin I_{1}$. For every $2 \leq i \leq n, x_{2} \cdots \cdots x_{n} \in I_{i}$ but $x_{1} \notin I_{i}$, so $x \notin I_{i}$. Thus $x \notin I_{1} \cup \cdots \cup I_{n}=R$, a contradiction.

Theorem 3.4 If a proper ring $R$ has a finite Goldie dimension, then every finitely generated ideal of $R$ consisting of zero-divisors has non-zero annihilator. 
Proof Since $R$ is of finite Goldie dimension it contains an essential ideal $I=I_{1} \oplus \cdots \oplus I_{n}$, where $I_{1}, \ldots, I_{n}$ are uniform ideals of $R$. Let $J=<a_{1}, a_{2}, \ldots, a_{k}>$ be an ideal generated by $a_{1}, a_{2}, \ldots, a_{k} \in R$ consisting of zero-divisors. Since $J$ consists of zero-divisors, for every $x \in J$, $a n_{R}(x) \neq 0$. Obviously $\operatorname{ann}_{R}(x)$ is an ideal of $R$, so essentiality of $I$ implies that $I \cap a n n_{R}(x) \neq 0$. Thus there are $x_{i} \in I_{i}$, not all equal 0 , such that $\left(x_{1}+\cdots+x_{n}\right) x=0$. However $x_{i} x \in I_{i}$, so $x_{i} x=0$ for all $1 \leq i \leq n$. This shows that if $x_{i} \neq 0$, then $x \in \bar{I}_{J i}$. Consequently $J=\bar{I}_{J 1} \cup \cdots \cup \bar{I}_{J n}$. Applying Lemmas 3.2 and 3.3 we get that $J=\bar{I}_{J i}$ for some $1 \leq i \leq n$. In particular $a_{1}, \ldots, a_{k} \in \bar{I}_{J i}$, so $a n n_{R}\left(a_{j}\right) \cap I_{i} \neq 0$ for $1 \leq j \leq k$. However $I_{i}$ is a uniform ideal of $R$, so $T=\operatorname{ann}_{R}\left(a_{1}\right) \cap \cdots \cap \operatorname{ann}_{R}\left(a_{k}\right) \cap I_{i} \neq 0$. Clearly $T \subseteq \operatorname{ann}_{R}\left(\left\{a_{1}, \ldots, a_{k}\right\}\right)$ and we are done.

Acknowledgments The authors thank the two referees for their valuable suggestion, which allowed to improve an earlier version of this paper. They also thank Professor Z. Bartosiewicz for calling their attention to the question and Professor E. R. Puczyłowski for his many useful comments on this work. Supported by Bialystok University of Technology Grant No. S/WI/2/11.

Open Access This article is distributed under the terms of the Creative Commons Attribution License which permits any use, distribution, and reproduction in any medium, provided the original author(s) and the source are credited.

\section{References}

1. Atiyah, M.F.; Macdonald, I.G.: Introduction to Commutative Algebra, Addison, Wesley Publishing Co., Reading, Mass. London Don Mills, Ont. (1969)

2. Bartosiewicz, Z.: Density of images of semigroup operators for linear neutral functional differential equations. J. Differ. Equ. 38, 161-175 (1980)

3. Bartosiewicz, Z.: Approximate controllability of neutral systems with delays in control. J. Differ. Equ. 51, 295-325 (1984)

4. Glaz, S.: Controlling the zero divisors of a commutative ring. Lecture Notes in Pure and Appl. Math, vol. 231, pp. $191-212$. Marcel Dekker, New York (2002)

5. Goldie, A.W.: The Structure of Noetherian Rings. Lectures on Rings and Modules, Lecture Notes in Mathematics. vol. 246, Springer, New York (1972)

6. Grzeszczuk, P.; Puczylowski, E.R.: On Goldie and dual Goldie dimensions. J. Pure Appl. Algebra 31(1-3), 47-54 (1984)

7. Hamilton, T.D.; Marley, T.: Non-Noetherian Cohen-Macaulay rings. J. Algebra 307, 343-360 (2007)

8. Huckaba, J.A.; Keller, J.M.: Annihilation of ideals in commutative rings. Pac. J. Math. 83(2), 375-379 (1979)

9. Huckaba, J.A.: Commutative Rings with Zero Divisors. Marcel Dekker Inc., New York (1988)

10. Lam, T.Y.: Lectures on Modules and Rings. Graduate Texts in Mathematics. vol. 189, Springer, New York (1999)

11. Lucas, T.G.: Two annihilator conditions: property (A) and (A.C.). Commun. Algebra 14, 557-580 (1986)

12. Lucas, R.G.: The diameter of a zero divisor graph. J. Algebra 301, 174-193 (2006)

13. Oman, G.; Schwiebert, R.: Rings which admit faithful torsion modules. Commun. Algebra (to appear)

14. Oman, G.; Schwiebert, R.: Rings which admit faithful torsion modules II. J. Algebra Appl. (to appear)

15. Quentel, Y.: Sur la compacité du spectre minimal d'un anneau. Bull. Soc. Math. France 99, 265-272 (1971)

16. Vasconcelos, W.V.: Annihilators of modules with a finite free resolution. Proc. Am. Math. Soc 29(3), 440-442 (1971) 